\title{
Terbinafine Preferred Antifungal with a Focus on Dermatophytes (A Review)
}

\author{
Mayank Panday1*, Divya Pandey², Prashant Upadhyay² and Sukirti \\ Upadhyay $^{2}$ \\ ${ }^{1}$ School of Pharmaceutical Science, IFTM University, Moradabad, India \\ ${ }^{2}$ School of Pharmacy, Oriental university, Indore, India \\ *Corresponding Author: Mayank Panday, School of Pharmaceutical Science, IFTM \\ University, Moradabad, India.
}

Received: May 04, 2020

Published: June 24, 2020

(C) All rights are reserved by Mayank

Panday., et al.

\begin{abstract}
17 years ago, Terbinafine was hailed in the global drug market to use as antifungal. In the treatment of superficial dermatophytosis terbinafine is become the first choice of drug, because of its effective mode of action, pharmacologic action and microbiologic profiles. Appropriate use of terbinafine as a topical and systemic drug needs to be used with appropriate guidelines. Terbinafine is primarily indicated and also discussed a contraindication for the treatment of non-dermatophyte infections. Terbinafine act by inhibiting the enzyme squalene epoxidase which is an important component of fungal cell membrane resulting in disintegration of fungal cell was allowing terbinafine to exert its fungicidal action. As per the recent advancement significant clinical relevance seen in activity of terbinafine when used in combination of other antifungal leads to decrease in resistance. This article reviews mode of action, antimycotic spectrum and disposition profile of terbinafine. we have also done a comparative analysis of terbinafine over other antifungals (griseofulvin, itraconazole, fluconazole) in the management of dermatophytes infection.
\end{abstract}

Keywords: Tinea; Trichophyton; Microsporum; Allylamine; Antifungal

\section{Introduction}

In 2008, the Oral terbinafine market has completed 12 years in the United States and 17 years globally. Terbinafine is sold in India as Terboderm by Omega Pharma and Tyza (Abbott Healthcare), Terbinafine first became available in Europe in 1991 and in the United States in 1996. The U.S. Food and Drug Administration has approved the first generic versions of prescription Lamisil (terbinafine hydrochloride) tablets. Since its launch, terbinafine has marked the first choice of all slots among oral and topical formulations. It is estimated to have captured nearly $80 \%$ of the greater than US $\$ 1.5$ billion worldwide onychomycosis market (the reason for making up to the majority of prescription for children's) [1,2]. In current situations of fungal infections terbinafine remains the only commercially available orally allylamine and shares the topical allylamine/benzylamine market with naftifine, butenafine and amorolfine. In past few years several new formulations have been added to the portfolio of this antimycotic group of treatment. In September 2007 the US Food and Drug Administration also approved an oral granule in the paediatric age group. With the formulation which is available in the non-US market globally like systemic formulation and topical solution.
This article will review the data on the mycology and pharmacology of terbinafine including its mode of action, antimycotic spectrum, disposition profile and therapeutic efficacy. The primary focus will surround dermatophytosis with a brief discussion on the role of terbinafine in dermatophyte infections with the guidelines for appropriate dosage uses.

Superficial infections are mainly caused by keratinophilic fungi (keratin loving fungi) they are present on our stratum corneum nails, and hair. Trichophyton, Epidermophyton, and Microsporum species. Tinea corporis and tinea cruris is the dermatophytosis of glabrous skin and groin.

In localized infection, topical preparation is the preferred formulation which has good bioavailability and penetration for the localized area. They do not affect the first pass metabolism and kill the fungal infections on topical sites and reduce the side effect and chances of drug-drug interactions. It helps patient's compliance, increases chances of good outcomes [3].

Among all the antifungals the terbinafine has chosen to the first line of treatment because of its unique pharmacological and My- 
cology action. It inhibits the squalene epoxidase, thereby inhibiting ergosterol action. In the past few years, the humid and hot climate helpful for the cause of dermatophytes infection and the terbinafine is consistently active against antifungals with a $90 \%$ fungicidal rate in $250 \mathrm{mg}$ once daily dose of terbinafine up to 2 weeks $[3,4]$. Currently, there are clinical failures and relapse cases are higher with terbinafine $[5,6]$. One of the principle reasons in the relapse of antifungals are decreased when the concentration increase in some relapse cases of dermatophytes infection up to $500 \mathrm{mg}$ once daily $[7,8]$.

Itraconazole is a group of antifungals which comes in an azole group and this drug molecule inhibit the cytochrome P450 enzyme and convert in lanosterol to ergosterol under demethylation. A 100 mg up to 2 weeks dose of itraconazole shows a significant reduction in dermatophytosis infection and higher concentration is required up to 7 days for the desired result and due to relapse cases $[9,10]$. In short interval dermatologists and physicians start using $200 \mathrm{mg}$ once daily for a prolonged period $[11,12]$.

Resistance of antifungal agents has been occurred widespread and used in conventional-dose with an increase in relapse rates promoting a need to find an effective first-line antifungal drug with lesser resistance and with a decrease in relapse rates. Hence, the present study was conducted to compare the efficacy of oral terbinafine versus itraconazole in the treatment of tinea corporis and tinea cruris.

\section{Clinical mycology}

Terbinafine discovered in 1983 and it is a member of the allylamine antifungals group. Due to the presence of tert-butyl acetylene substitution of the phenyl ring on the side chain of the molecule. The efficacy of oral terbinafine is increased 100 times in in-vitro studies of Naftifine $[13,14]$. Terbinafine interfere in the biosynthesis of fungi. By inhibiting the squalene epoxidase, it will stop the formation of ergosterol, with the help of these step squalene convert into 2,3-oxidosqualene (an ergosterol precursor). so ultimately the synthesis is stopped in lack of ergosterol and lead a cell death because of weak cell wall integrity. In-vitro study states that the terbinafine has minimal drug interaction and a small concentration inhibit 95\% activity of squalene epoxidase [15-17].

In the clinical cases of dermatophytes terbinafine outcomes are observed and it found that the terbinafine is showing susceptibility to some organism including pathogenic yeast, thermally dimorphic fungi [18]. On other hand Terbinafine showing a wonderful activity in a reduction of some species, Trichophyton, Microsporum and Epidermophyton.
The minimum inhibitory concentration (MIC) of Drug terbinafine has observed very lower to kill the fungi including other species of dermatophytes comparatively with other antifungals which are also active against these organisms like triazoles, imidazoles, griseofulvin $[19,20]$.

While resistance is very rare but the increased cases of antifungals show that the cross-resistance when it used with different antifungals [21]. When the terbinafine is used as a combination in the management of invasive mycoses. Against Aspergillus fumigatus, then indifference was observed that the combination of terbinafine and amphotericin. B, similarly, fluconazole, itraconazole including terbinafine did not improve the activity of $A$. fumigatus. Moreover, combination of triazole and terbinafine shows a synergy response (greater effect) on this pathogen [22]. In the management of invasive cases terbinafine showed an ultimate utility and it starts using as a most important agent.

\section{Clinical pharmacology}

Terbinafine is 70 to $80 \%$ absorbed from oral route and it shows a linear absorption towards the ideal dose (250 $\mathrm{mg}$ ) of terbinafine and the total body exposure is directly proportional to dose. The percentage of absorption dose doesn't appear from children and defer in adults it always based on the per kilogram on body weight [25]. If the children are less than 6 years old it will give according to its body weight, and not recommended in less than 2 years old.

Topical based terbinafine cream and gel formulation absorption having a normal range of skin is 746 to $949 \mathrm{ng} / \mathrm{cm}$. Within 7 days of application the concentration is increased in stratum corneum by $15 \%$ moreover AUC is also increased upto $40 \%$ under 1 week. It has been observed that in stratum corneum the topical preparation is well absorbed the resultant systemic exposure is several orders of magnitude lower than observed after oral terbinafine administration (Table 1 and 2).

Topical preparation is commonly used in the treatment of dermatophytes and the good bioavailability shows a great reduction in fungi, topical is used as the first line of therapy in the superficial skin infection. A great bioavailability and Efficacy of topical preparation will help to reduce the longer time of treatment. Moreover, the topical cream and gel sand other preparation are helping to minimize the side effect and chances of recurrence and increase therapeutic response [26].

\section{Therapeutic uses}

Terbinafine is recommended in the management and treatment of dermatophytosis (tinea cruris, capitis and tinea Corporis) and 


\begin{tabular}{|l|c|c|c|c|c|}
\hline \multicolumn{1}{|c|}{ Parameter } & $\begin{array}{c}\text { Adults 125 mg } \\
\text { single-dose (n= 26) }\end{array}$ & $\begin{array}{c}\text { Adults 250 mg } \\
\text { single-dose (n= 29) }\end{array}$ & $\begin{array}{c}\text { Adults 125 mg } \\
\text { steady-state (n = 10) }\end{array}$ & $\begin{array}{c}\text { Adults 250 mg } \\
\text { steady-state (n = 22) }\end{array}$ & $\begin{array}{c}\text { Children 125 mg } \\
\text { single-dose (n = 28) }\end{array}$ \\
\hline Tmax (h) & $1.3-1.5$ & $1.4-1.5$ & 1.6 & 1.2 & $1.7-2.1$ \\
\hline Cmax (ng/mL) & $506-565$ & $1340-1656$ & 646 & 1700 & $706-909$ \\
\hline AUC (h*ng/mL) & $1624-2135$ & $4740-6762$ & 3720 & 10481 & $2967-4104$ \\
\hline Cl/F (L/h/kg) & 1.2 & - & 0.55 & - & 1.9 \\
\hline Vss/F (L/kg) & 19.2 & - & - & & 19.5 \\
\hline $\mathrm{t} 1 / 2 \alpha(\mathrm{h})$ & 0.7 & 0.35 & - & & 1.2 \\
\hline $\mathrm{t} 1 / 2 \beta(\mathrm{h})$ & 26.7 & $12.6-14.2$ & - & 396 & 14.7 \\
\hline $\mathrm{t} 1 / 2 \gamma(\mathrm{h})$ & - & - & - & & 156 \\
\hline
\end{tabular}

Table 1: Pharmacokinetic parameter estimated of terbinafine in oral administration.

\begin{tabular}{|c|c|c|}
\hline Parameter & 1\% gel $\times \mathbf{7}$ days healthy skin & 1\% cream 7 days \\
\hline Stratum corneum Cmax $\left(\mu \mathrm{g} / \mathrm{cm}^{2}\right)$ & 0.91 & $0.94-2$ \\
\hline Stratum corneum AUC $\left(\mathrm{h}^{*} \mu \mathrm{g} / \mathrm{cm}^{2}\right)$ & 12.7 & $11.7-13.5$ \\
\hline Tissue $\mathrm{t} 1 / 2(\mathrm{~h})$ & 1.2 & 68 \\
\hline Plasma Cmax $(\mathrm{ng} / \mathrm{mL})$ & 3.82 & - \\
\hline Plasma AUC $\left(\mathrm{h}^{*} \mathrm{ng} / \mathrm{mL}\right)$ & 63 & - \\
\hline
\end{tabular}

Table 2: Local exposure of topical terbinafine application.

onychomycosis. Moreover, terbinafine is using on other pathogens including systemic mycoses other than the dermatophytes. Explore the utility of terbinafine to other infections, orally administered tablets of terbinafine are not effective on Pityriasis versicolor.

\section{Cutaneous dermatophytes}

There are number of species present in today's environment which is responsible to cause dermatophytes infection on face, groin, trunk, feet. For the treatment of these body areas topically will be the first line and if the case is found under widespread or chronic in nature systemic therapy will be preferable $[27,28]$.

Tinea cruris: Is called as jock itch and it is most common in males due to occlusive garments, $1 \%$ terbinafine hydrochloride cream, gel formulation has shown a significant reduction on a lesion and impact on a mycological cure rate by $94 \%$ and clinically cure rate by $84 \%$ with this overall ranging rate upto $63 \%$ to $83 \%$. Statically topical use of terbinafine gives an effective result in the management of fungal infection. Then a 2 - week treatment of $2 \%$ ketoconazole cream [29-33].

Tinea pedis: Due to the lack of sebaceous gland secretion over sole and its web spaces make them very favorable to such infection. Reason of leading chronicity of infection are increased sweating, occlusive footwear use of laden socks and the major causative pathogen which is responsible for tinea pedis are, T. rubrum, $T$. mentagrophytes and E. floccosum. 1\% terbinafine cream, gel formulation was applied on the infected area and comparatively mycological cure rate $82 \%$ to $97 \%$ and efficacy rate $64 \%$ to $86 \%$.

\section{Onychomycosis}

In simple words onychomycosis is an infection of the nail caused by dermatophyte, mold or yeast. Tinea ungunium is also referred to as dermatophyte infection of nail. Various clinical patterns of invasion are present in onychomycosis. The most common organisms are T. mentagrophytes and T. rubrum, and distal lateral subungual onychomycosis, the most common clinical type. So, in the treatment of onychomycosis includes both oral and topical with combination therapy of terbinafine and itraconazole as a pulse dosing gives satisfactory results with a period of 12 to 24 weeks in cases of fingernail or toenail. Respectively, in all the nail problems one-half is accounted by finger or toenail onychomycosis in this fungal infection nail became thickened, discoloured, or prone to splitting. Toenail infections are mostly caused by dermatophytes whereas over $50 \%$ of cases are caused by non-dermatophytes species [34-36].

Commercially available group of antifungals like griseofulvin, Terbinafine, itraconazole, are used in the management of such infections and it's efficacy is also dependent on the duration of course which is typically required in the treatment. There is a study rate of 12 months which is described below and the protected growth rate of nail including which drug remains in the affected nail. Af- 
ter approval, numerous of studies are performed and evaluated based on dose regimens for the finger nail, toenail fungal infection with terbinafine $250 \mathrm{mg} /$ day. With this dose clinical rate is $44 \%$ to $77 \%$ and Mycologic rate is $72 \%$ to $92 \%$ was recorded, respectively. Notably, there is a small difference found in patients who are treated in 12, 18 and 24 weeks. Moreover, fingernail onychomycosis comparatively shows a good response rate of $71 \%$ to $100 \%$ [37-42] when the daily dose of terbinafine $250 \mathrm{mg}$ once in a day was given with topical preparation of amorolfine once daily gives more improvement in fungal infection response [43-47]. When compared with $500 \mathrm{mg}$ administered daily for 1 week (followed by 3 weeks off) for the treatment of distal subungual onychomycosis, traditional dosing again proved superior to intermittent dosing. Mycological cure of the target toenail (71\% vs 59\%); clinical cure of the target toenail ( $45 \%$ vs $29 \%$ ); complete cure of the target toenail ( $40 \%$ vs $28 \%$ ); and complete cure of all 10 toenails $(25 \%$ vs $15 \%$ ) were all statistically greater with standard dosing. No significant differences in complete cure have been observed based on the number of pulses administered; however, a clear trend is noted with response rates increasing steadily from one to four pulses. As noted with traditional dosing, higher cure rates were observed for fingernails treated with pulse- dosing as compared with toe nails. Mycological and clinical cure rates were $89 \%$ and $72 \%$ for dermatophytes, albeit lower (67\%) for infections caused by yeast. As expected, based on the comparative data generated from traditional dosing trials, the combination of pulse therapy with ancillary topical therapy does not substantially improve outcome over treatment with terbinafine alone [48-51].

\section{Non-dermatophyte infection}

In the management of non- dermatophytosis infection the higher MIC is despite to pathogenic yeast. Topical application of terbinafine appears in the management of pityriasis versicolor and mainly caused by Malassezia furfur. Terbinafine is an orally administered terbinafine (250 mg twice daily) has been used successfully for the treatment of cutaneous candidiasis. Moreover, a summary and additional [52,53].

\section{Adverse effect}

In the treatment of dermatophyte, Terbinafine $250 \mathrm{mg} /$ day evaluated with very low Chances of adverse drug reactions. In the clinical evaluation and on efficacy parameter systemic terbinafine in children and adults have noted as adverse event rate upto $52 \%$ and $10 \%$ with attribute with the drug. Most common adverse events are gastrointestinal irritation, nausea, vomiting, abdominal pain, weight gain, headache when higher doses are administered [61-66].

\begin{tabular}{|c|c|c|c|c|}
\hline Organism & Dose Rate & Site & $\begin{array}{c}\text { Recover } \\
\text { Percentage }\end{array}$ & $\begin{array}{c}\text { Refer- } \\
\text { ence }\end{array}$ \\
\hline $\begin{array}{l}\text { Sporothrix } \\
\text { schenckii }\end{array}$ & $\begin{array}{c}250 \text { mg twice a } \\
\text { day upto 8-36 } \\
\text { weeks }\end{array}$ & $\begin{array}{c}\text { Cutaneous/ } \\
\text { subcutane- } \\
\text { ous }\end{array}$ & Success & {$[59,60]$} \\
\hline Piedra hortae & $\begin{array}{c}250 \mathrm{mg} / \text { day } \\
\text { upto } 6 \text { weeks }\end{array}$ & Scalp & Effective & [58] \\
\hline $\begin{array}{l}\text { Fonsecaea } \\
\text { monophora }\end{array}$ & $\begin{array}{l}250 \mathrm{mg} / \text { day } \\
\text { upto } 10 \text { weeks }\end{array}$ & Skin & Cured & [57] \\
\hline \begin{tabular}{|l|} 
Cladosporium \\
carrionii
\end{tabular} & $\begin{array}{l}500 \mathrm{mg} / \text { day- } \\
\text { upto } 12 \text { months }\end{array}$ & Skin & $\begin{array}{c}83 \% \text { to } 100 \\
\%\end{array}$ & {$[55,56]$} \\
\hline $\begin{array}{l}\text { Aspergillus } \\
\text { sydowii }\end{array}$ & $\begin{array}{c}500 \mathrm{mg} / \text { day- } 3 \\
\text { months }\end{array}$ & Toenail & Failed & [51] \\
\hline $\begin{array}{l}\text { Aspergillus } \\
\text { spp }\end{array}$ & $\begin{array}{l}500 \mathrm{mg} / \text { day- } 3 \\
\text { months }\end{array}$ & Toenail & $\begin{array}{c}88 \% \text { clinical } \\
\text { and Myco- } \\
\text { logical }\end{array}$ & [54] \\
\hline
\end{tabular}

\section{Table}

The liver and hematologic system are most commonly involving rate of $0.04 \%$, Hepatotoxicity ranging and the liver failure are the consequences which is very rarely reported with Terbinafine. Only $2.2 \%$ of patient has treated with Terbinafine has experienced the changes in liver function test [67]. Notably, a singular trial report comparing terbinafine with griseofulvin for the treatment of tinea capitis. a change of eating habits in $4.7 \%$ and $5.5 \%$ of children, respectively. Whether, however, this was due to changes in taste perception is unknown [54]. Of note, fewer patients receiving terbinafine pulse therapy as compared with traditional dosing experience elevations in liver enzymes or taste disturbances; however, the overall percentage of patients discontinuing therapy for adverse events was comparable between dosing strategies [68].

With the use of terbinafine various ocular disorders have been observed with the use of terbinafine in a patient after two weeks the bilateral anterior optic neuropathy with decreased vision and optic disc edema was reported (500 mg/day). After discontinuing the medication, the vision improved. After 12 days of therapy Anterior uveitis was reported in a second patient with acquired immune deficiency syndrome. As in the previous case, symptoms resolved with discontinuation of terbinafine [69-71].

Among patients treated with topical terbinafine preparations, adverse events are primarily restricted to mild to moderate local skin reactions which may occur in as many as $6 \%$ of patients [72].

\section{Dermatophytosis treatment}

Treatment of recalcitrant dermatophytosis is dependent on their condition which are as follows: 
- $\quad$ MIC level against skin pathogen.

- $\quad$ Secretion profile of drug.

- Concentration achieved at target site.

In the cases of dermatophytosis or recalcitrant dermatophytosis if the patient is not responding may be they have some immunity issue so the therapy will be planned accordingly. Which is provide in below figure [73].

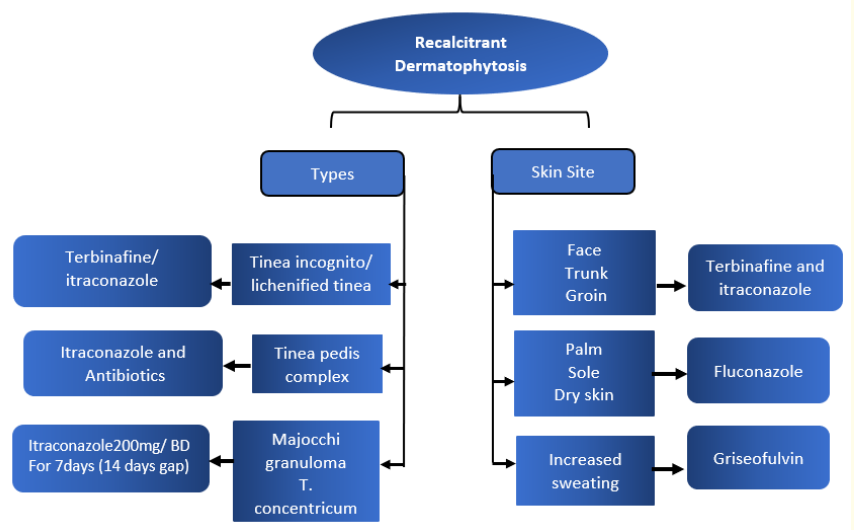

Figure

\section{Conclusion}

In the cases of recalcitrant dermatophytosis Terbinafine is a preferred antifungal choice due to its fungicidal action of skin and nail and because of its characteristics to present in stratum corneum, sebum nails and hair for months after stopping the medication is likely responsible for the better treatment of dermatophytes and its kill the fungi at the same MIC and MFC so chances of drug to drug interactions are likely less and a preferred Choice for poly pharmacy patients. And numerous clinical trials are corroborating the suitability and efficacy are far better than the existing antifungals. While the treatment in dermatophytosis or a systemic mycosis has been limited to date, Moreover Terbinafine is still remain as a first line of treatment in most of the dermatophytosis infections $250 \mathrm{mg}$ once daily is sufficient as per the IADVL guidelines. Infrequent but the severe adverse effect (Hepatotoxicity) and potential for the drug interactions for the medication that rely on CYP2D6 as a primary route of metabolism.so twice daily Terbinafine upto 4 week gives 80 to $100 \%$ result and its more effective than placebo in mycological cure and as well as clinical outcomes and reduces the risk of symptoms score, and over all efficacy.

\section{Bibliography}

1. Brown M and Traynor M. "Treatment of fungal nail infections". Drug Delivery Market Research Reports (2007): 17-19.

2. Suh DC., et al. "Usage patterns of medical services and prescription drugs in patients with tinea capitis". Journal of the American Academy of Dermatology 50 (2004): 86.

3. Moodahadu-Bangera LS., et al. "Eberconazole-pharmacological clinical review". Indian Journal of Dermatology, Venereology and Leprology 78 (2012): 217-222.

4. Singh S and Beena PM. "Comparative study of different microscopic techniques and culture media for the isolation of dermatophytes". Indian Journal of Medical Microbiology 21 (2003): 21-24.

5. Newland JG and Abdel-Rahman SM. "Update on terbinafine with a focus on dermatophytosis". Clinical, Cosmetic and Investigational Dermatology 2 (2009): 49-63.

6. McClellan KJ., et al. "Terbinafine. An update of its use in superficial mycoses". Drugs 58 (1999): 179-202.

7. Osborne CS., et al. "Amino acid substitution in Trichophyton rubrum squalene epoxidase associated with resistance to terbinafine". Antimicrobial Agents and Chemotherapy 49 (2005): 2840-2844.

8. Majid I., et al. "Relapse after oral terbinafine therapy in dermatophytosis: A Clinical and mycological study". Indian Journal of Dermatology 61 (2016): 529-533.

9. Sanglard D. "Emerging threats in antifungal-resistant fungal pathogens". Frontiers in Medicine 3 (2016): 11.

10. Babu PR., et al. "Efficacy and safety of terbinafine $500 \mathrm{mg}$ once daily in patients with dermatophytosis". Indian Journal of Dermatology 62 (2017): 395-399.

11. Ardeshna KP., et al. "Successful treatment of recurrent dermatophytosis with isotretinoin and itraconazole". Indian Journal of Dermatology 82 (2016): 579-582.

12. Donckar PD., et al. "Itraconazole: What clinicians should know”. Indian Journal of Dermatology 3 (2017): 4-10.

13. Ryder NS and Dupont MC. "Inhibition of squalene epoxidase by allylamine antimycotic compounds. A comparative study of the fungal and mammalian enzymes". Biochemical Journal 230.3 (1985): 765-770. 
14. Ganzinger U., et al. "Allylamines: topical and oral treatment of dermatomycoses with a new class of antifungal agents". Acta Dermato-Venereologica (Stockh). 121 (1986): 155-160.

15. Ryder NS. "Specific inhibition of fungal sterol biosynthesis by SF 86-327, a new allylamine antimycotic agent". Antimicrobial Agents and Chemotherapy 27.2 (1985): 252-256.

16. Birnbaum JE. "Pharmacology of the allylamines". Journal of the American Academy of Dermatology 23.4-2 (1990): 782-785.

17. Ryder NS. "Terbinafine: mode of action and properties of the squalene epoxidase inhibition". British Journal of Dermatology 126.39 (1992): 2-7.

18. Venugopal PV and Venugopal TV. "Antidermatophytic activity of allyl-amine derivatives". Indian Journal of Pathology and Microbiology 37.4 (1994): 381-388.

19. Venugopal PV and Venugopal TV. "Antidermatophytic activity of garlic (Allium sativum) in vitro". International Journal of Dermatology 34.4 (1995): 278-279.

20. Da Silva Barros ME., et al. "Evaluation of susceptibility of Trichophyton mentagrophytes and Trichophyton rubrum clinical isolates to antifungal drugs using a modified CLSI microdilution method (M38-A)". Journal of Medical Microbiology 56.4 (2007): 514-518.

21. Kohli A Smriti., et al. "In vitro low-level resistance to azoles in Candida albicans is associated with changes in membrane lipid fluidity and asymmetry". Antimicrobial Agents and Chemotherapy 46.4 (2002): 1046-1052.

22. Ryder NS and Leitner I. "Activity of terbinafine against Aspergillus in vitro, in combination with amphotericin B or triazoles (Abstract E54)". 36 ${ }^{\text {th }}$ Interscience Conference on Antimicrobial Agents and Chemotherapy (1996): 15-18.

23. Gupta AK., et al. "Antifungal agents: an overview. Part II". Journal of the American Academy of Dermatology 30.6 (1994): 911-933.

24. Jensen JC. "Clinical pharmacokinetics of terbinafine (Lamisil)”. Clinical and Experimental Dermatology 14.2 (1989): 110-113.

25. Abdel-Rahman SM., et al. "Pharmacokinetics of terbinafine in young children treated for tinea capitis". The Pediatric Infectious Disease Journal 24.10 (2005): 886-891.
26. Moodahadu-Bangera LS., et al. "Eberconazole-pharmacological and clinical review". Indian Journal of Dermatology, Venereology and Leprology 78 (2012): 217-222.

27. Odom R. "Pathophysiology of dermatophyte infections". Journal of the American Academy of Dermatology 28.5-1 (1993): S2-S7.

28. Farag A., et al. "One-week therapy with oral terbinafine in cases of tinea cruris/corporis". British Journal of Dermatology 131.5 (1994): 684-686.

29. Evans EG., et al. "Short-duration therapy with terbinafine 1\% cream in dermatophyte skin infections". British Journal of Dermatology 130.1 (1994): 83-87.

30. Bonifaz A and Saul A. "Comparative study between terbinafine $1 \%$ emulsion-gel versus ketoconazole $2 \%$ cream in tinea cruris and tinea corporis". The European Journal of Dermatology 10.2 (2000): 107-109.

31. Budimulja U., et al. "Once daily treatment with terbinafine $1 \%$ cream (Lamisil) for one week is effective in the treatment of tinea corporis and cruris. A placebo-controlled study". Mycoses 44.7-8 (2001): 300-306.

32. Lebwohl M., et al. "Efficacy and safety of terbinafine $1 \%$ solution in the treatment of interdigital tinea pedis and tinea corporis or tinea cruris". Cutis 67.3 (2001): 261-266.

33. Zaias N., et al. "Efficacy of a 1-week, once-daily regimen of terbinafine $1 \%$ cream in the treatment of tinea cruris and tinea corporis". Journal of the American Academy of Dermatology 29.4 (1993): 646-648.

34. Scher RK. "Onychomycosis is more than a cosmetic problem". British Journal of Dermatology 130.43 (1994): 15.

35. Degreef H. "Onychomycosis". British Journal of Clinical Practice 71 (1990): 91-97.

36. Midgley G., et al. "Mycology of nail disorders". Journal of the American Academy of Dermatology 31.3-2 (1994): S68-S74.

37. Baudraz-Rosselet F., et al. "Treatment of onychomycosis with terbinafine". British Journal of Dermatology 126.39 (1992): 4046. 
38. Farkas B., et al. "Terbinafine (Lamisil) treatment of toenail onychomycosis in patients with insulin-dependent and noninsulin-dependent diabetes mellitus: a multicentre trial". British Journal of Dermatology 146.2 (2002): 254-260.

39. Goodfield MJ., et al. "Short term treatment of dermatophyte onychomycosis with terbinafine". British Medical Journal 304.6835 (1992): 1151-1154.

40. Pollak R and Billstein SA. "Efficacy of terbinafine for toenail onychomycosis. A multicenter trial of various treatment durations". Journal of the American Podiatric Medical Association 91.3 (2001): 127-131.

41. Watson A., et al. "Terbinafine in onychomycosis of the toenail: a novel treatment protocol". Journal of the American Academy of Dermatology 33.5-1 (1995): 775-779.

42. Zaias N and Serrano L. "The successful treatment of finger Trichophyton rubrum onychomycosis with oral terbinafine". Clinical and Experimental Dermatology 14.2 (1989): 120-123.

43. Albanese G., et al. "Short therapy for tinea unguium with terbinafine: four different courses of treatment". Mycoses 38.5-6 (1995): 211-214.

44. Jennings MB., et al. "Treatment of toenail onychomycosis with oral terbinafine plus aggressive debridement: IRON-CLAD, a large, randomized, open-label, multi- center trial". Journal of the American Podiatric Medical Association 96.6 (2006): 465473.

45. Avner S., et al. "Combination of oral terbinafine and topical ciclopirox compared to oral terbinafine for the treatment of onycho- mycosis". Journal of Dermatological Treatment 16.5-6 (2005): 327-330.

46. Baran R. "Topical amorolfine for 15 months combined with 12 weeks of oral terbinafine, a cost-effective treatment for onychomycosis". British Journal of Dermatology 145.60 (2001): 15-19.

47. Baran R., et al. "A multicentre, randomized, controlled study of the efficacy, safety and cost-effectiveness of a combination therapy with amorolfine nail lacquer and oral terbinafine compared with oral terbinafine alone for the treatment of onychomycosis with matrix involvement". British Journal of Dermatology 157.1 (2007): 149-157.
48. Warshaw EM., et al. "Pulse versus continuous terbinafine for onycho- mycosis: a randomized, double-blind, controlled trial". Journal of the American Academy of Dermatology 53.4 (2005): 578-584.

49. Nakano N., et al. "Combination of pulse therapy with terbinafine tablets and topical terbinafine cream for the treatment of dermatophyte onychomycosis: a pilot study". The Journal of Dermatology 33.11 (2006): 753-758.

50. Jaiswal A., et al. "An open randomized comparative study to test the efficacy and safety of oral terbinafine pulse as a monotherapy and in combination with topical ciclopirox olamine $8 \%$ or topical amorolfine hydrochloride $5 \%$ in the treatment of onychomycosis". Indian Journal of Dermatology, Venereology and Leprology 73.6 (2007): 393-396.

51. Takahata Y., et al. "Treatment of dermatophyte onychomycosis with three pulses of terbinafine (500 mg day for a week)". Mycoses 52.1 (2009): 72-76.

52. Faergemann J., et al. "A double-blind comparison of levels of terbinafine and itraconazole in plasma, skin, sebum, hair and nails during and after oral medication". Acta Dermato-Venereologica 77.1 (1997): 74-76.

53. Jung EG., et al. "Systemic treatment of skin candidosis: a randomized comparison of terbinafine and ketoconazole". Mycoses 37.9-10 (1994): 361-365.

54. Gianni C and Romano C. "Clinical and histological aspects of toenail onychomycosis caused by Aspergillus spp: 34 cases treated with weekly intermittent terbinafine". Dermatology 209.2 (2004): 104-110.

55. Esterre P., et al. "Treatment of chromomycosis with terbinafine: preliminary results of an open pilot study". British Journal of Dermatology 134.46 (1996): 33-36.

56. Xibao Z., et al. "Treatment of chromoblastomycosis with terbinafine: a report of four cases". Journal of Dermatological Treatment 16.2 (2005): 121-124.

57. Zhang J., et al. "Successful treatment for chromoblastomycosis caused by Fonsecae monophona: a report of three cases in Guangdong, China". Mycoses 52.2 (2009): 176-181. 
58. Gip L. “Terbinafine for black piedra”. Lancet 341.8853 (1993): 1164.

59. Coskun B., et al. "Sporotrichosis successfully treated with terbinafine and potassium iodide: case report and review of the literature". Mycopathologia 158.1 (2004): 53-56.

60. Hull PR and Vismer HF. "Treatment of cutaneous sporotrichosis with terbinafine". British Journal of Dermatology 126.39 (1992): 51-55.

61. Elewski BE., et al. "Terbinafine hydrochloride oral granules versus oral griseofulvin suspension in children with tinea capitis: results of two randomized, investigator-blinded, multicenter, international, controlled trials". Journal of the American Academy of Dermatology 59.1 (2008): 41-54.

62. Finlay AY. "Global overview of Lamisil". British Journal of Dermatology 130.43 (1994): 1-3.

63. Pollak R and Billstein SA. "Safety of oral terbinafine for toenail onycho-mycosis". Journal of the American Podiatric Medical Association 87.12 (1997): 565-570.

64. Villars V and Jones TC. "Clinical efficacy and tolerability of terbinafine (Lamisil)--a new topical and systemic fungicidal drug for treatment of dermatomycoses". Clinical and Experimental Dermatology 14.2 (1989): 124-127.

65. De Backer M., et al. "Twelve weeks of continuous oral therapy for toenail onychomycosis caused by dermatophytes: a double-blind comparative trial of terbinafine $250 \mathrm{mg}$ /day versus itraconazole 200 mg/day". Journal of the American Academy of Dermatology 38.5-3 (1998): S57-S63.

66. Drake LA., et al. "Oral terbinafine in the treatment of toenail onychomycosis: North American multicenter trial". Journal of the American Academy of Dermatology 37.5-1 (1997): 740745.

67. Oral antifungal treatments for superficial dermatophytosis and onychomycosis: a meta-analysis". The American Journal of Medicine 120.9 (2007): 791-798.

68. Sigurgeirsson B., et al. "Intermittent versus continuous terbinafine in the treatment of toenail onychomycosis: a randomized, double-blind comparison". Journal of Dermatological Treatment 17.1 (2006): 38-44.
69. Gupta AK., et al. "The development of green vision in association with terbinafine therapy". Archives of Dermatological Research 132.7 (1996): 845-846.

70. Price T., et al. "Anterior uveitis in a patient with AIDS who was treated with terbinafine for oral candidiasis: a potential druginduced reaction". Clinical Infectious Diseases 25.3 (1997): 752-753.

71. Yulek F., et al. "Bilateral anterior optic neuropathy associated with use of terbinafine". Clinical and Experimental Ophthalmology 36.5 (2008): 488-489.

72. Schopf R., et al. "Efficacy and tolerability of terbinafine $1 \%$ topical solution used for 1 week compared with 4 weeks clotrimazole $1 \%$ topical solution in the treatment of interdigital tinea pedis: a randomized, double- blind, multi-centre, 8-week clinical trial". Mycoses 42.5-6 (1999): 415-420.

73. Sardana et al. "IADVL manual on management of dermatophytosis". First Edition. cbs publisher and distributor (2018).

\section{Assets from publication with us}

- Prompt Acknowledgement after receiving the article

- Thorough Double blinded peer review

- Rapid Publication

- Issue of Publication Certificate

- High visibility of your Published work

Website: https://www.actascientific.com/

Submit Article: https://www.actascientific.com/submission.php

Email us: editor@actascientific.com

Contact us: +919182824667 